\title{
Adding feasibility constraints to a ranking rule under a monotonicity constraint
}

\author{
Raúl Pérez-Fernández, ${ }^{1,2}$ Michael Rademaker, ${ }^{2}$ Pedro Alonso, ${ }^{1}$ Irene Díaz, ${ }^{3}$ Bernard De Baets ${ }^{2}$ \\ ${ }^{1}$ Department of Mathematics, University of Oviedo, Spain \\ ${ }^{2}$ KERMIT, Department of Mathematical Modelling, \\ Statistics and Bioinformatics, Ghent University, Belgium \\ ${ }^{3}$ Department of Computer Science, University of Oviedo, Spain
}

\begin{abstract}
We propose a new point of view in the long-standing problem where several voters have expressed a linear order relation (or ranking) over a set of candidates. For a ranking $a>b>c$ to represent a group's opinion, it would be logical that the strength with which $a>c$ is supported should not be less than the strength with which either $a>b$ or $b>c$ is supported. This intuitive property can be considered a monotonicity constraint, and has been addressed before. We extend previous approaches in the following way: as the voters are expressing linear orders, we can take the number of candidates between two candidates to be a measure of the degree to which one candidate is preferred to the other. In this way, intensity of support is both counted as the number of voters who indicate $a>c$ is true, as well as the distance between $a$ and $c$ in these voters' rankings. The resulting distributions serve as input for a natural ranking rule that is based on stochastic monotonicity and stochastic dominance. Adapting the previous methodology turns out to be non-trivial once we add some natural feasibility constraints.
\end{abstract}

Keywords: Group Decision Making, Linear Order, Weak Order, Monotonicity, Stochastic Dominance, Integer Linear Programming.

\section{Introduction}

Probably the most simple and ancient ranking rule is plurality, where the candidate with the most votes is declared the winner. Yet, it is not the most widely used method, as it suffers from a multitude of issues. Arrow's impossibility theorem has shown that there will always be some issues with any ranking rule, as a number of intuitive properties are mutually exclusive [3]. In fact, two of the most widely known voting rules are explicitly divided on the issue of "should a pairwise majority winner automatically be the winner of an election?": Condorcet's practical method [6] and the Borda count [4] answer this question in diametrically opposed ways.

In 1982, Riker [12] realized that the same set of votes can yield different winners, depending on the voting rule used. Furthermore, he showed that, in some extreme cases, even every candidate in an election can be elected the winner according to some voting rule. In order to do so, he studied the notion of consensus and demonstrated that most ranking rules can be characterized as measuring the distance from this consensus by a metric. For example, consider the least reversals method by Condorcet, which consists of looking for an acyclic pairwise majority relation, disregarding the pairwise majorities with smaller support if needed. This idea can be thought of as a way to minimize the distance to a consensus, an acyclic pairwise majority relation in this context. A deeper review of voting rules can be found in [5].

It will be no surprise that a big family of ranking rules consists of those that measure how close each possible linear order of the candidates comes to fulfilling a specific property $[1,2,5]$, for example, being in agreement with each pairwise majority. As an example of a recent such ranking rule, Rademaker and De Baets proposed in [9] a natural application in social choice of their previous works on restoring monotonicity $[7,8,10,11]$. In [9], they propose a ranking rule that amounts to finding the ranking for which it holds that the votes are closest to satisfying a natural monotonicity property. They consider that, for a ranking $a>b>c$, monotonicity implies that the strength with which $a>c$ is supported should not be less than the strength with which either $a>b$ and $b>c$ is supported. Positional information is not explicitly taken into account in [9], and we propose to extend the method to take this hitherto unexploited information into account. After all, for a linear order $a>b>c$, we can observe that $b$ is in between $a$ and $c$, making the support for $a$ over $c$ stronger than that for $a$ over $b$, which is not considered in [9]. In order to make consistent use of this information, we employ some feasibility constraints.

\section{Problem setting}

Consider a set $C$ of $k$ candidates and let there be an unknown 'true' linear order $>$ between them. A set $R$ of $r$ voters, which are imprecise observers of $>$, is asked to compare the candidates according to their respective imprecise estimates regarding their 
suitability. These estimates are expressed as linear orders $\succ_{i}$.

Let us denote by $p_{i}(a, b) \in\{0,1\}$ the preference that voter $i$ has shown for candidate $a$ over candidate $b$. Let $p_{i}(a, b)=0$ denote that $b$ is preferred to $a$ and, analogously, $p_{i}(a, b)=1$ denote that $a$ is preferred to $b$. Of course, $p_{i}(a, b)=1-p_{i}(b, a)$, for any $a, b \in C$. Furthermore, as we are working with linear orders, $p_{i}$ is transitive.

Our goal is to look for the 'true' linear order considering the information provided by these voters.

Let $C_{*}^{2}=C^{2} \backslash\{(a, a) \mid a \in C\}$ and $r_{\succ}(a)$ be the position of candidate $a$ in the linear order $\succ$ (considering the greatest is position 1). Note that $r_{\succ}(a)=1+\sum_{\substack{b \in C \\ b \neq a}} p_{\succ}(b, a)$.

For any $i \in\{-k+1, \ldots,-1,1, \ldots, k-1\}$ and for any $(a, b) \in C_{*}^{2}$, we define:

$$
D_{j}^{i}(a, b)=\mathbb{1}\left(r_{\succ_{j}}(a)-r_{\succ_{j}}(b)=-i\right),
$$

where $\mathbb{1}(A)$ is the indicator function of the logical expression $A$, we further define:

$$
D^{i}(a, b)=\sum_{j=1}^{r} D_{j}^{i}(a, b) .
$$

With each couple $(a, b) \in C_{*}^{2}$ we can associate its distribution. A distribution is a function $D: C_{*}^{2} \rightarrow$ $\{0,1, \ldots, r\}^{2 k-2}$ defined via:

$D(a, b)=$

$=\left(D^{1-k}(a, b), \ldots, D^{-1}(a, b), D^{1}(a, b), \ldots, D^{k-1}(a, b)\right)$.

For instance, if we have three linear orders $\succ_{1}$ : $a>b>c>d, \succ_{2}: d>a>c>b$ and $\succ_{3}: a>$ $d>b>c$. We can observe that $D_{1}^{3}(a, d)=1$ (as in the first linear order $a$ is preferred to $d$ and there are two elements in between), $D_{2}^{-1}(a, d)=1$ (as in the second linear order $d$ is preferred to $a$ and there are no elements in between), $D_{3}^{1}(a, d)=1$ (as in the third linear order $a$ is preferred to $d$ and there are no elements in between) and $D_{j}^{i}(a, d)=0$ for the other values of $i$ and $j$. Therefore, joining all the experts, we will obtain $D^{-3}(a, d)=0, D^{-2}(a, d)=$ $0, D^{-1}(a, d)=1, D^{1}(a, d)=1, D^{2}(a, d)=0$ and $D^{3}(a, d)=1$. Finally, the obtained distribution for $(a, d)$ is $D(a, d)=(0,0,1,1,0,1)$.

These distributions have the following properties, commonly known as feasibility properties:

Proposition 1 Let $D$ be a distribution on a set of $k$ candidates $C$ based on a profile of $r$ voters. Then, $D$ satisfies the following properties:

- Completeness: for any $(a, b) \in C_{*}^{2}$ :

$$
\sum_{\substack{i=1-k \\ i \neq 0}}^{k-1} D^{i}(a, b)=r .
$$

- Reciprocity: for any $i \in\{-k+1, \ldots, k-1\}$ with $i \neq 0$ and any $(a, b) \in C_{*}^{2}$ :

$$
D^{i}(a, b)=D^{-i}(b, a) .
$$

- Stability: for any $i \in\{-k+1, \ldots, k-1\}$ with $i \neq 0$ :

$$
\sum_{(a, b) \in C_{*}^{2}} D^{i}(a, b)=r(k-|i|) .
$$

\section{- Peakedness:}

for any $a \in C$ and any $i \in\{2, \ldots, k-1\}$ :

$$
\begin{aligned}
& \sum_{b \in C} D^{i}(a, b) \leq \sum_{b \in C} D^{i-1}(a, b), \\
& \sum_{b \in C} D^{i}(b, a) \leq \sum_{b \in C} D^{i-1}(b, a) .
\end{aligned}
$$

for any $a \in C$ and any $i \in\{1-k, \ldots,-2\}$ :

$$
\begin{aligned}
& \sum_{b \in C} D^{i}(a, b) \leq \sum_{b \in C} D^{i+1}(a, b), \\
& \sum_{b \in C} D^{i}(b, a) \leq \sum_{b \in C} D^{i+1}(b, a) .
\end{aligned}
$$

We can define stochastic dominance $\unrhd$ with respect to a distribution $D$ over $C_{*}^{2}$. Let $(a, d)$ and $(b, c)$ be two couples in $C_{*}^{2}$.

$D(a, d) \unrhd D(b, c) \Leftrightarrow(\forall j)\left(\sum_{\substack{i=1-k \\ i \neq 0}}^{j} D^{i}(a, d) \leq \sum_{\substack{i=1-k \\ i \neq 0}}^{j} D^{i}(a, d)\right)$.

Trivially, to a linear order relation $>$ corresponds a total weak order relation $\geq$. Furthermore, a linear order relation $>$ gives rise to the following strict partial order relation $\sqsupset_{\uparrow}$ on $C_{*}^{2}$.

Definition 1 Let $C$ be a set of $k$ candidates (provided with a linear order $>)$. Then, a relation between two elements $(a, d),(b, c) \in C_{*}^{2}$ is defined in the following way:

$(a, d) \sqsupset_{\uparrow}(b, c) \Leftrightarrow a \geq b, c \geq d, b>c,(a>b$ or $c>d)$.

As $\sqsupset \uparrow$ is only satisfied by elements on the upper half of $C_{*}^{2}$, we will often use the following notation:

$$
C_{* \uparrow}^{2}=\left\{(a, b) \in C_{*}^{2} \mid a>b\right\} .
$$

In Figure 1 we can observe the diagram of $\sqsupset_{\uparrow}$ for a linear order $a>b>c>d$. Informally, we will call this diagram 'the triangle diagram'.

Furthermore, we can extend this relation to $C_{*}^{2}$.

Definition 2 Let $>$ be a linear order. It defines a strict partial order relation $\sqsupset$ on $C_{*}^{2}$ :

$$
(a, d) \sqsupset(b, c) \Leftrightarrow \begin{cases}(a, d) \sqsupset_{\uparrow}(b, c) & \text { if } b>c, \\ (c, b) \sqsupset_{\uparrow}(d, a) & \text { if } d>a, \\ (a, d) \sqsupset_{\uparrow}(c, b) & \\ (c, b) \sqsupset_{\uparrow}(a, d) & \text { if } a>d, c>b . \\ a=c, b=d & \end{cases}
$$




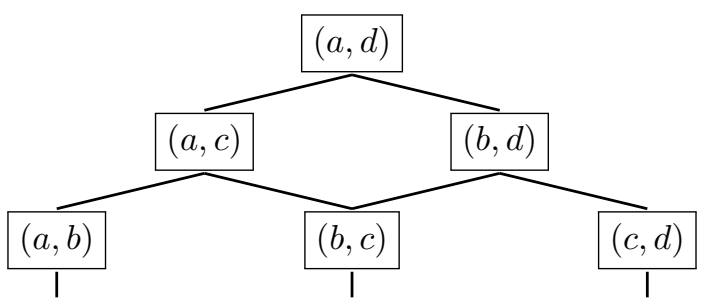

Figure 1: Graphical representation of $\sqsupset \uparrow$ for ranking $a>b>c>d$. Triangle diagram.

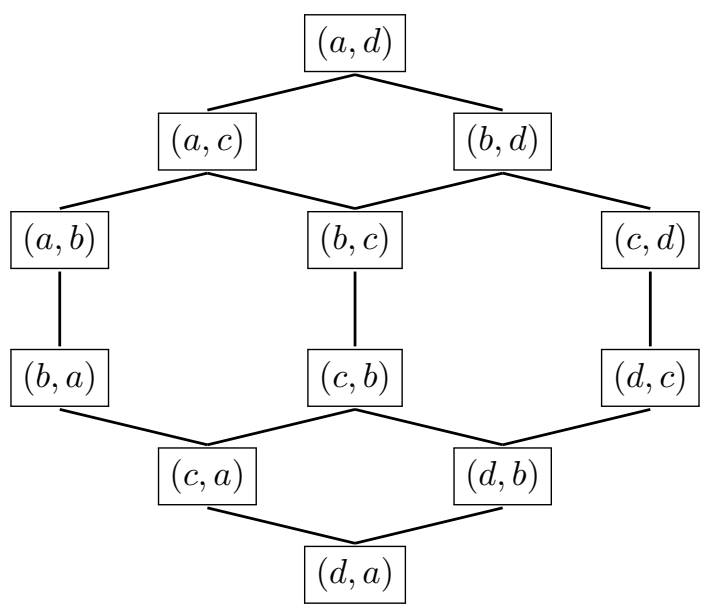

Figure 2: Graphical representation of $\sqsupset$ for ranking $a>b>c>d$. Diamond diagram.

In Figure 2 we show a graphical representation of $\sqsupset$ for a linear order $a>b>c>d$. Informally, we will call this diagram 'the diamond diagram'.

However, as we work with reciprocal distributions, the distributions of couples in the lower part of the diagram defined by $\sqsupset$ do not actually provide additional information beyond that provided by the top part. To exploit this knowledge, we always work with $\sqsupset \uparrow$.

Definition 3 Let $C$ be a set of $k$ candidates (provided with a linear order $>$ ) and the corresponding $C_{*}^{2}$ (provided with the strict partial order $\sqsupset$ ). Then a couple $(a, b) \in C_{*}^{2}$ is said to be a basic element of $C_{*}^{2}$ if $a \gg b$, i.e.

$$
a>b \text { and } \nexists c \text { such that } a>c>b \text {. }
$$

The set of all basic elements of $C_{*}^{2}$ is denoted by $\mathcal{B}\left(C_{*}^{2}\right)$.

Therefore, for a given ranking $>$, we can define the natural monotonicity constraint in the following way:

Definition 4 For a given linear order $>$ and corresponding $\sqsupset$, the monotonicity constraint on a distribution $D$ is the following: for any $(a, d),(b, c) \in C_{*}^{2}$ such that $(a, d) \quad \sqsupset_{\uparrow}$ $(b, c)$ :

$$
D(a, d) \unrhd D(b, c),
$$

and for any $(e, f) \in \mathcal{B}\left(C_{*}^{2}\right)$ :

$$
D(e, f) \unrhd D(f, e) .
$$

$D$ is called monotone if these constraints are fulfilled.

This natural monotonicity constraint does not need to be satisfied by every distribution $D$, as the voters could disagree in their comparisons. However, it seems natural to require that the winning ranking satisfies this property or at least that it is not too far from satisfying it. When this monotonicity constraint is not satisfied by any ranking, the goal will be to look for a distribution $D^{\prime}$ that is monotone with relation to at least one ranking. Obviously, among all the possible monotone distributions, we want the closest to the observed one.

Therefore, it is necessary to introduce at this moment a distance metric between distributions.

$$
\begin{aligned}
d\left(D, D^{\prime}\right) & =\sum_{(a, b) \in C_{* \uparrow}^{2}} d\left(D(a, b), D^{\prime}(a, b)\right), \text { where } \\
d\left(D(a, b), D^{\prime}(a, b)\right)= & \\
= & \sum_{\substack{i=1-k \\
i \neq 0}}^{k-1}\left|\sum_{\substack{j=1-k \\
j \neq 0}}^{i} D^{j}(a, b)-D^{\prime j}(a, b)\right| .
\end{aligned}
$$

When looking for the closest monotone distribution, we want to focus on the distributions that also satisfy the feasibility properties introduced in Proposition 1. In this case, we are working with distributions that do not need to satisfy these properties, but we want to impose them as constraints. Therefore, the whole set of feasibility properties (reciprocity, completeness, stability and peakedness) will be named feasibility constraints from now on.

Definition 5 For a given distribution $D$ and $a$ ranking $>$, a closest monotone distribution (under feasibility constraints) $D^{\prime}: C_{*}^{2} \rightarrow\{0,1, \ldots, r\}^{2 k-2}$ for a given distance metric $d$ is one satisfying:

(MON) For any $(a, d) \sqsupset(b, c): D^{\prime}(a, d) \unrhd D^{\prime}(b, c)$

(REC) For any $(a, b)$ and any $i$ :

$$
D^{\prime i}(a, b)=D^{\prime-i}(b, a)
$$

(COM) For any $(a, b): \sum_{\substack{i=1-k \\ i \neq 0}}^{k-1} D^{\prime i}(a, b)=r$

(STA) For any $i: \sum_{(a, b) \in C_{*}^{2}} D^{\prime i}(a, b)=r(k-|i|)$ 
(PEA) For any $a \in C$ and any $i \in\{2, \ldots, k-1\}$ :

$$
\sum_{b \in C} D^{\prime i}(a, b) \leq \sum_{b \in C} D^{i-1}(a, b)
$$

For any $a \in C$ and any $i \in\{1-k, \ldots,-2\}$ :

$$
\sum_{b \in C} D^{\prime i}(a, b) \leq \sum_{b \in C} D^{\prime i+1}(a, b)
$$

(OPT) There is not another distribution $D^{\prime \prime}$ : $C_{*}^{2} \rightarrow\{0,1, \ldots, r\}^{2 k-2}$ satisfying the above conditions while

$$
d\left(D, D^{\prime \prime}\right)<d\left(D, D^{\prime}\right)
$$

Finding a closest monotone distribution is important in order to find an optimal linear order:

Definition 6 For a given distribution D, an optimal linear order $>$ (with a corresponding closest monotone distribution $\left.D^{\prime}\right)$ is a linear order for which it holds that there exists no ranking $>^{\prime}$ (with a corresponding closest monotone distribution $D^{\prime \prime}$ ) such that $d\left(D, D^{\prime \prime}\right)<d\left(D, D^{\prime}\right)$.

In a previous work [9], Rademaker and De Baets were able to calculate the "number of changes needed in order to impose monotonicity w.r.t. $\succ$ " by solving a flow network problem. However, when we add condition (STA), we are no longer able to use a flow network representation. Instead, we will need to solve an integer linear programming problem for each possible linear order. We feel adding (STA) is worthwhile, as it follows the "reassigning votes" rationale from [9] much closer than in the original paper. Now, when changing a vote that compared extremes in certain ranking (at distance $k-1$ ), another couple of candidates will have to become extremes.

The number of unknown values and the number of constraints will depend on the number $k$ of candidates:

- Number of (positive integer) variables: $2 k(k-1)^{2}$.

- Monotonicity constraints (inequalities): $4(k-1)^{2}(k-2)+2(k-1)^{2}$.

- Reciprocity constraints (equalities): $k(k-1)^{2}$.

- Completeness constraints (equalities): $k(k-1)$.

- Stability constraints (equalities): $2(k-1)$.

- Peakedness constraints (inequalities): $4 k(k-2)$.

However, because of completeness and reciprocity, some of these constraints become superfluous. We can focus on $\sqsupset_{\uparrow}$ (triangle diagram) instead of $\sqsupset$. This yields the following constraints:

- Number of (positive integer) variables: $k(k-1)^{2}$.
- Monotonicity constraints (inequalities): $2(k-1)^{2}(k-2)+(k-1)^{2}$

- Completeness constraints (equalities): $\frac{k(k-1)}{2}$.

- Stability constraints (equalities): $(k-1)$.

- Peakedness constraints (inequalities): $2 k(k-2)$.

More formally, the integer linear programming problem we will need to solve is then:

minimize $\quad d\left(D, D^{\prime}\right)$ subject to:

- for any $j \in\{1-k, \ldots,-1,1, \ldots, k-2\}$, for any $(a, d) \sqsupset_{\uparrow}(b, c)$,

$$
\sum_{\substack{i=1-k \\ i \neq 0}}^{j} D^{\prime i}(a, d) \leq \sum_{\substack{i=1-k \\ i \neq 0}}^{j} D^{i}(b, c)
$$

- for any $j \in\{1-k, \ldots,-1\}$, for any $(e, f) \in \mathcal{B}\left(C_{*}^{2}\right)$,

$$
\sum_{\substack{i=1-k \\ i \neq 0}}^{j} D^{i}(e, f) \leq \sum_{\substack{i=1-k \\ i \neq 0}}^{j} D^{\prime-i}(e, f)
$$

- for any $(a, b) \in C_{* \uparrow}^{2}$,

$$
\sum_{\substack{i=1-k \\ i \neq 0}}^{k-1} D^{\prime i}(a, b)=r
$$

- for any $i \in\{1, \ldots, k-1\}$,

$$
\sum_{(a, b) \in C_{* \uparrow}^{2}} D^{\prime i}(a, b)+D^{\prime-i}(a, b)=r(k-i)
$$

- for any $a \in C$ and any $i \in\{2, \ldots, k-1\}$,

$$
\begin{aligned}
& \sum_{\substack{b \in C \\
a>b}} D^{\prime i}(a, b)+\sum_{\substack{b \in C \\
b>a}} D^{\prime-i}(a, b) \leq \\
& \leq \sum_{\substack{b \in C \\
a>b}} D^{\prime i-1}(a, b)+\sum_{\substack{b \in C \\
b>a}} D^{\prime-i}(a, b)
\end{aligned}
$$

- for any $a \in C$ and any $i \in\{1-k, \ldots,-2\}$,

$$
\begin{aligned}
\sum_{\substack{b \in C \\
a>b}} D^{\prime i}(a, b)+\sum_{\substack{b \in C \\
b>a}} D^{\prime-i}(a, b) \leq \\
\quad \leq \sum_{\substack{b \in C \\
a>b}} D^{\prime i+1}(a, b)+\sum_{\substack{b \in C \\
b>a}} D^{\prime-1-i}(a, b)
\end{aligned}
$$

- for any $i \in\{1-k, \ldots,-1,1, \ldots, k-1\}$, for any $(a, b) \in C_{* \uparrow}^{2}$.

$$
D^{\prime i}(a, b) \in \mathbb{Z} \cap[0, r]
$$

Remark 1 It must be noted that $d\left(D, D^{\prime}\right)$ is a sum of absolute values and we will have an integer nonlinear programming problem (INLP) instead of an integer linear programming problem (ILP). Fortunately, such absolute values can easily be handled by adding auxiliary variables to our problem. 
Minimize $\sum_{i=1}^{n}\left|z_{i}\right|$ (with $z_{i}$ a linear combination of a vector of unknown values $x$ ) under certain conditions $A x \leq b$ is equivalent to:

$$
\begin{aligned}
& \text { Minimize } \sum_{i=1}^{n} y_{i} \text { under the following conditions: } \\
& A x \leq b \\
& z_{i} \leq y_{i} \\
& \text { for any } i=1, \ldots, n \text {, } \\
& -z_{i} \leq y_{i} \\
& \text { for any } i=1, \ldots, n \text {, } \\
& y_{i} \geq 0 \\
& \text { for any } i=1, \ldots, n \text {. }
\end{aligned}
$$

\section{Allowing for weak orders}

In real problems, some of the candidates can be similarly worthy and be considered equivalent. To model this kind of problems we will need to allow for weak orders instead of linear orders as winning rankings. Remember that weak orders are binary relations satisfying reflexivity and transitivity (but not antisymmetry).

In our particular problem, we can intuitively observe that if two elements $a$ and $b$ are equivalent then, for every different element $c$, the distributions $D(a, c)$ and $D(b, c)$ will need to be quite similar. Furthermore, $D(a, b)$ and $D(b, a)$ will need to coincide too. Therefore, it seems intuitive to add to our integer linear programming problem of Eq. (1) these constraints.

Formally, adding the equivalence $a \sim b$ (where $\left.(a, b) \in \mathcal{B}\left(C_{*}^{2}\right)\right)$, the constraints would be:

$$
\begin{aligned}
& \text { for any } j \in\{1-k, \ldots,-1,1, \ldots, k-1\}, \\
& \text { for any } c \in C \backslash\{a, b\}, \\
& \sum_{\substack{i=1-k \\
i \neq 0}}^{j} D^{i}(a, c)=\sum_{\substack{i=1-k \\
i \neq 0}}^{j} D^{\prime i}(b, c) \\
& \text { for any } j \in\{1-k, \ldots,-1\}, \\
& \sum_{\substack{i=1-k \\
i \neq 0}}^{j} D^{\prime i}(a, b)=\sum_{\substack{i=1-k \\
i \neq 0}}^{j} D^{\prime-i}(a, b)
\end{aligned}
$$

However, a technical problem involving the number of voters may arise in this part of the study. In a hypothetical case where alternatives $a$ and $b$ are equivalent, we would need to have that $D(a, b)=$ $D(b, a)$. However, if we have an odd number of voters this condition is not going to be satisfied because $D(a, b)$ and $D(b, a)$ are symmetric by definition of distribution.

This uncomfortable issue can be solved considering a threshold $t$ and changing the preceding constraints to the following one:

$$
\begin{aligned}
& \sum_{c \in C \backslash\{a, b\}} \sum_{\substack{i=1-k \\
j \neq 0}}^{k-1}\left|\sum_{\substack{i=1-k \\
i \neq 0}}^{j} D^{i}(a, c)-\sum_{\substack{i=1-k \\
i \neq 0}}^{j} D^{i}(b, c)\right|+ \\
& +\sum_{j=1-k}^{-1}\left|\sum_{\substack{i=1-k \\
i \neq 0}}^{j} D^{i}(a, b)-\sum_{\substack{i=1-k \\
i \neq 0}}^{j} D^{-i}(a, b)\right| \leq t .
\end{aligned}
$$

Note that, by monotonicity, the absolute values in this equation can be removed considering the right side of the absolute value.

As we want to minimize this threshold $t$, we will need to solve an equivalent minimization problem where we will treat firstly the minimization of $t$ and secondly the optimization of $d\left(D, D^{\prime}\right)$ :

minimize $\delta \cdot t+d\left(D, D^{\prime}\right)$ s.t. constraints (1) and (2) where $\delta$ is an upper bound of $d\left(D, D^{\prime}\right)$.

When adding constraints to a linear programming problem, the optimal value of the objective function will get worse (or remain equal). This makes it difficult to compare the number of changes needed to obtain a suitable weak order to the number needed to obtain a suitable linear order. A possible solution to this problem is to output the Pareto optimal front, i.e. the set of the closest weak orders with each number of equivalence classes, which includes the linear order with $k$ singleton equivalence classes. We provide a straightforward algorithm in order to obtain the Pareto optimal front.

Algorithm 1 INPUT: Profile $\mathcal{R}$ with $r$ voters and $k$ candidates

OUTPUT: Pareto optimal front $\mathcal{P}$

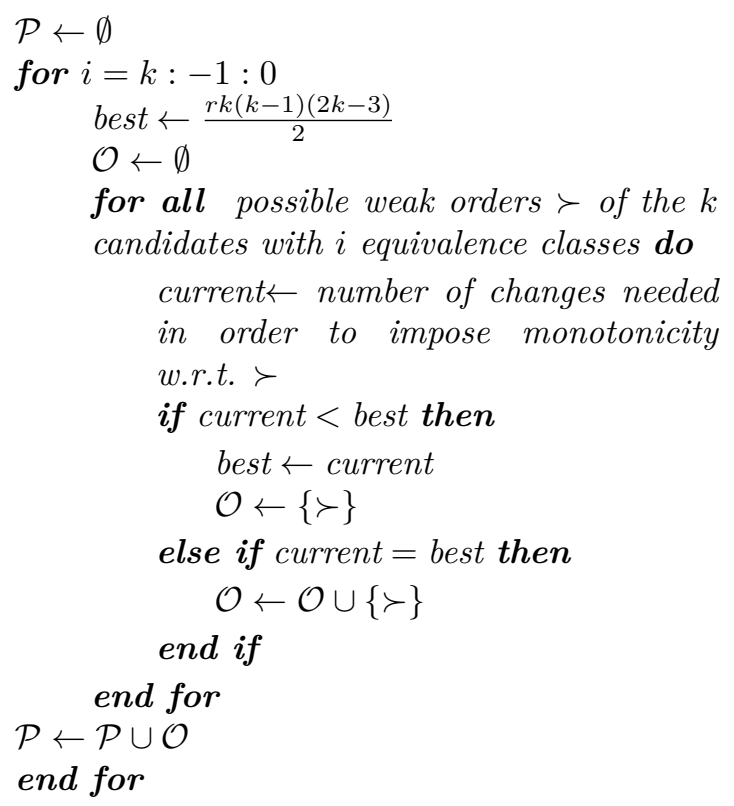

\section{Remark 2 Pruning technique}

The number of weak orders of the $k$ candidates with $i$ equivalence classes scales rapidly and can 
cause computational issues. Consequently, every possible reduction of the number of elements to be analysed is of interest. A simple way to do so is by using lower bounds: once we have obtained the costs of each linear order, we can use these obtained values in order to obtain a lower bound of each weak order. The idea is that if the closest linear order has a cost of $c_{0}$ changes and the best weak order with $k-1$ equivalence classes has a cost of $c_{1}$ (obviously $c_{0} \leq c_{1}$ ), we can discard every weak order with $k-1$ equivalence classes with a linear extension of cost greater than $c_{1}$.

\section{Case study and computational example}

The methodology is applied to a dataset coming from a decision making problem concerning management of the Lar rangeland in Iran, an area of great local economic, ecological and social importance. When drawing up new management plans, the Iranian government asked 31 representatives of different stakeholder groups to rank four plans according to their perceived suitability on different criteria. We represent the data corresponding to the Wildlife Diversity Criterion here. For more details, we refer to [13].

\begin{tabular}{c|c} 
frequency & ranking \\
\hline 18 & $a \succ b \succ c \succ d$ \\
3 & $c \succ d \succ b \succ a$ \\
3 & $d \succ c \succ b \succ a$ \\
2 & $b \succ a \succ c \succ d$ \\
1 & $b \succ c \succ a \succ d$ \\
1 & $b \succ d \succ c \succ a$ \\
1 & $c \succ b \succ d \succ a$ \\
1 & $d \succ b \succ c \succ a$ \\
1 & $b \succ c \succ d \succ a$
\end{tabular}

Table 1: Expressed rankings and their frequency for the Wildlife Criterion in the Lar rangeland decision problem.

In Table 1 we can see the linear orders given by the experts in the Lar rangeland decision problem. We can observe that a majority of voters have expressed a preference for $a \succ b \succ c \succ d$. However, as our considered ranking rule is not plurality, the winning ranking could differ from the most voted linear order.

The goal right now will be to look for the optimal linear order. Remember that our notion of consensus is the linear order that is the closest to satisfy the monotonicity property. In Table 2 the number of changes needed to restore monotonicity for every linear order is shown. We can conclude that $a \succ b \succ c \succ d$ is the closest linear order with a cost of 45 . Therefore, we are going to consider it as the optimal linear order.

In Figure 3 we can observe the original distribution and a monotone relabelling with 45 changes.

\begin{tabular}{l|c||c|c} 
ranking & changes & ranking & changes \\
\hline$a \succ b \succ c \succ d$ & 45 & $c \succ a \succ b \succ d$ & 97 \\
$a \succ b \succ d \succ c$ & 90 & $c \succ a \succ d \succ b$ & 132 \\
$a \succ c \succ b \succ d$ & 80 & $c \succ b \succ a \succ d$ & 89 \\
$a \succ c \succ d \succ b$ & 125 & $c \succ b \succ d \succ a$ & 131 \\
$a \succ d \succ b \succ c$ & 119 & $c \succ d \succ a \succ b$ & 141 \\
$a \succ d \succ c \succ b$ & 130 & $c \succ d \succ b \succ a$ & 146 \\
$b \succ a \succ c \succ d$ & 60 & $d \succ a \succ b \succ c$ & 136 \\
$b \succ a \succ d \succ c$ & 83 & $d \succ a \succ c \succ b$ & 147 \\
$b \succ c \succ a \succ d$ & 78 & $d \succ b \succ a \succ c$ & 139 \\
$b \succ c \succ d \succ a$ & 106 & $d \succ b \succ c \succ a$ & 148 \\
$b \succ d \succ a \succ c$ & 118 & $d \succ c \succ a \succ b$ & 152 \\
$b \succ d \succ c \succ a$ & 125 & $d \succ c \succ b \succ a$ & 153
\end{tabular}

Table 2: Closest linear orders.

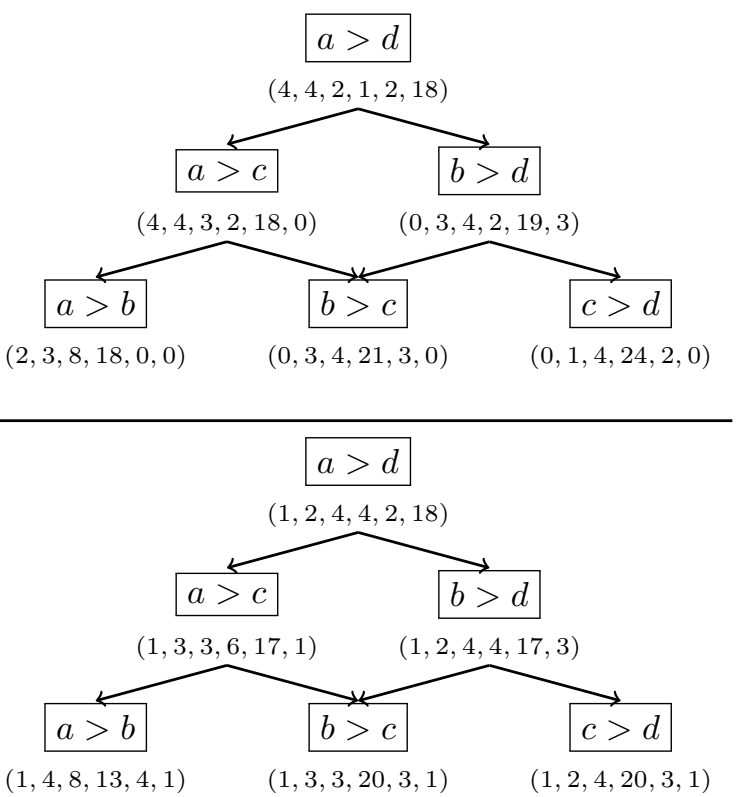

Figure 3: Original data (top) and monotone relabelling (bottom).

Next, we determine the Pareto optimal front. We already know that $a \succ b \succ c \succ d$ is the closest linear order to monotonicity so it belongs to the Pareto optimal front. In addition, we trivially know that $a \sim b \sim c \sim d$ belongs to the Pareto front too. Therefore, we will need to look for the closest weak orders with 3 and 2 equivalence classes.

Considering the closest linear order $a \succ b \succ c \succ$ $d$, we are going to check the number of changes needed to restore monotonicity for the three possible weak orders with 3 equivalence classes that are linearly extended by $a \succ b \succ c \succ d$ :

$$
\begin{aligned}
& \text { - } a \sim b \succ c \succ d: 77 ; \\
& \text { - } a \succ b \sim c \succ d: 83 ; \\
& \text { - } a \succ b \succ c \sim d: 91 \text {. }
\end{aligned}
$$

Note that the best weak order with 3 equivalence classes that is linearly extended by $a \succ b \succ c \succ d$ is $a \sim b \succ c \succ d$. We need now to check if there is another weak order with 3 equivalence classes (not 
necessarily linearly extended by $a \succ b \succ c \succ d$ ) with a smaller number of changes. Normally, we would need to check all the possible weak orders but, using the pruning technique, we can reduce the number of studied weak orders.

Consulting Table 2, we can observe that the only linear order with a cost smaller than 77 is $b \succ a \succ$ $c \succ d$. Therefore, we only need to study the three possible weak orders with 3 equivalence classes that are linearly extended by $b \succ a \succ c \succ d$ :

- $b \sim a \succ c \succ d: 78$;

- $b \succ a \sim c \succ d: 98$;

- $b \succ a \succ c \sim d: 92$.

Note that the costs of $a \sim b \succ c \succ d$ and $b \sim$ $a \succ c \succ d$ differ in a unit. This is due to the odd number of voters that renders a real tie between both alternatives impossible.

We can also note that the calculation of the costs of $b \succ a \sim c \succ d$ and $b \succ a \succ c \sim d$ is unnecessary. $b \succ c \succ a \succ d$ is a linear extension of the first one and it has a cost of $78(\geq 77)$. Analogously, $b \succ a \succ d \succ c$ is a linear extension of the second one and it has a cost of $83(\geq 77)$.

We can therefore observe that $a \sim b \succ c \succ d=$ $b \sim a \succ c \succ d$ is the closest weak order with three equivalence classes and it belongs to the Pareto optimal front.

Finally, we are going to calculate the costs of the weak orders with 2 equivalence classes linearly extended by $a \succ b \succ c \succ d$.

- $a \succ b \sim c \sim d$ : 132;

- $a \sim b \succ c \sim d: 95$;

- $a \sim b \sim c \succ d: 112$.

The linear orders with a cost smaller than 95 are $a \succ b \succ c \succ d(45), a \succ b \succ d \succ c(90), a \succ c \succ$ $b \succ d(80), b \succ a \succ c \succ d(60), b \succ a \succ d \succ c$ (83), $b \succ c \succ a \succ d(78)$ and $c \succ b \succ a \succ d(89)$.

Therefore, the possible weak orders with 2 equivalence classes obtained from this linear orders are:

- $a \succ b \sim c \sim d: 132$;

- $a \sim b \succ c \sim d: 95$;

- $a \sim b \sim c \succ d: 112$;

- $a \sim b \sim d \succ c: 146$

- $a \sim c \succ b \sim d: 140$;

- $b \succ a \sim c \sim d: 128$;

- $b \sim c \succ a \sim d: 129$;

- $c \succ a \sim b \sim d: 145$.

Note that it is not necessary to compute all these costssince we can find linear extensions with a cost higher than 95. Finally, we add $a \sim b \succ c \sim d$ to the Pareto optimal front, yielding:

$$
\begin{aligned}
& \mathcal{P}=\{a \succ b \succ c \succ d, \quad a \sim b \succ c \succ d, \\
& a \sim b \succ c \sim d, \quad a \sim b \sim c \sim d\} .
\end{aligned}
$$

\section{Conclusions and open problems}

In this work, we extended an existing ranking method to take into account hitherto unused information, guarantee additional intuitive properties (the feasibility conditions, first and foremost) and to allow for more flexibility on the output. Due to the non-trivial nature of the extension, an integer linear problem needed to be constructed. The use of lower bounds to speed up computation has been illustrated, and the full Pareto front of possible optimal linear and weak order solutions has been computed in an example application. Weak order solutions are important in practice, as, often, multiple candidates can be considered equally suited.

Nevertheless, it remains an open problem to decide when such an equivalence is justified. To determine when this is the case, one will probably have to examine the inputs to the ranking method. Identifying quantifiable indicators that point to an equivalence being appropriate, is a challenging task. On a related note, allowing voters to express equivalence between candidates is another interesting area of future research. This will probably require a new distance metric, among other things. Making sure voters who express a linear order are not penalized or advantaged with respect to those who express a weak order is a topic that requires careful consideration.

\section{Aknowledgements}

This work has been partially supported by Campus of International Excellence of University of Oviedo.

\section{References}

[1] N.G. Andjigaa, A.Y. Mekukob and Issofa Moyouwoua, Metric rationalization of social welfare functions. Mathematical Social Sciences, 72, 14 23, 2014.

[2] V. Conitzer and T. Sandholm, Common voting rules as maximum likelihood estimators. In Proceedings of the 21st Conference on Uncertainty in Artificial Intelligence, Edinburgh, 145-152, 2005.

[3] P. Fishburn, Paradoxes of voting. American Political Science Review, 68(2), 537-546, 1974.

[4] I. McLean and A. Urken, Classics of Social Choice, University of Michigan Press, Ann Arbor, 1995 .

[5] T. Meskanen and H. Nurmi, Closeness Counts in Social Choice. In Power, Freedom, and Voting, Springer, 289-306, 2008.

[6] E.J. Nanson, Methods of election. Transactions and Proceedings of the Royal Society of Victoria XIX, 197-240, 1882. Reprinted in I. McLean and A. Urken, 1995.

[7] M. Rademaker and B. De Baets, Aggregation of monotone reciprocal relations with application 
to group decision making. Fuzzy Sets and Systems, 184(1), 29-51, 2011.

[8] M. Rademaker and B. De Baets, Optimal restoration of stochastic monotonicity with respect to cumulative label frequency loss functions. Information Sciences, 181(4), 747-757, 2011.

[9] M. Rademaker and B. De Baets, A ranking procedure based on a natural monotonicity constraint. Information Fusion, 17, 74-82, 2014.

[10] M. Rademaker, B. De Baets and H. De Meyer, Loss optimal monotone relabeling of noisy multi-criteria data sets. Information Sciences, 179(24), 4089-4096, 2009.

[11] M. Rademaker, B. De Baets and H. De Meyer, Optimal monotone relabelling of partially nonmonotone ordinal data. Optimization Methods and Software, 27(1), 17-31, 2012.

[12] W.H. Riker, Liberalism against populism: A Confrontation between the Theory of Democracy and the Theory of Social Choice. In San Francisco: W.H. Freeman, 1982.

[13] K. Zendehdel, M. Rademaker, B. De Baets and G. Van Huylenbroeck, Environmental decision making with conflicting social groups: A case study of the Lar rangeland in Iran. Journal of Arid Environments, 74(3), 349-402, 2010. 